\title{
L-Band Antenna Array for Next Generation DLR Airborne SAR Sensor
}

\author{
Diego Lorente, Markus Limbach, Bernd Gabler \\ SAR-Technology, Microwaves and Radar Institute, German Aerospace Center \\ Münchener Str. 20, 82234 Weßling, Germany \\ diego.lorentecatalan@dlr.de
}

\begin{abstract}
The Microwaves and Radar Institute at the German Aerospace Center (DLR) operates an airborne versatile multi-frequency SAR sensor (F-SAR). Due to the applications of remote-sensing imaging at low frequencies, a new dual polarized L-Band antenna system for the next generation sensor is presented in this paper. Despite the restricted size of the used aircraft, the new antenna optimizes the available space in the antenna carrier by providing more antenna elements, in comparison with the current L-Band sensor, without increasing the inter-element mutual coupling and thereby enhancing the radiation properties of the antenna.

Keywords - phased array, remote-sensing application, Synthetic Aperture Radar (SAR), airborne sensor, planar antenna.
\end{abstract}

\section{INTRODUCTION}

The Microwaves and Radar Institute of the German Aerospace Center (DLR) plays a key role in the field of remote sensing, not only for spaceborne applications such as the worldwide well-known missions of TanDEM-X and TerraSAR-X but also for airborne sensors.

For the increasing demand and promising applications of Synthetic Aperture Radar (SAR), airborne SAR provides an interesting scope of research for remote sensing applications as it supports future spaceborne missions such as TanDEM-L, Biomass, Sentinel and others.

Airborne SAR is employed by the DLR to study signal processing concepts, to experiment with innovative methods and techniques, and to develop and test components and hardware [1].

Due to the penetrating nature of electromagnetic waves for lower frequencies and by means of SAR interferometry, remote sensing from flying platforms provides a useful way to monitor forest density (deforestation study), tree height (tomography), as well as to perform glaciology studies for the measurement of snow and ice layer thicknesses and soil moisture, among other applications.

In addition, SAR polarimetry can be applied by using polarization diversity to identify different scattering mechanisms.

In order to exploit the capabilities of Synthetic Aperture Radar at lower frequency bands, a new antenna system in L-Band for the next generation of the DLR airborne SAR sensor (F-SAR) is presented, which also can be relevant to the upcoming spaceborne mission TanDEM-L.

Despite the always restricted available area in the airplane for the antenna installation, the new antenna system maximizes the number of elements in comparison with the last generation sensor. This improvement enhances significantly the radiation properties of the whole antenna by allowing to shape more accurately the radiation pattern. A special effort has to be made regarding the antenna compaction and the inter-element coupling reduction, in order to exploit the most the limited available space for the antenna.

This work will be structured as following: first of all, the system requirements for the next generation airborne sensor will be mentioned. Consequently, the design of a single dual polarized antenna element is presented and the required steps in order to achieve the desired performance are explained. This design will be validated by means of comparing the simulation results with the measurement of a real manufactured antenna. Later, this single antenna design is extended and used to build a linear array of five identical elements with amplitude and phase distribution in order to shape the radiation pattern in elevation as required. The antenna array is also manufactured and measured. Finally, the conclusion and future work are discussed.

\section{SySTEM REQUIREMENTS}

To fulfill the requirements and cover its wide range of applications, a dual polarized antenna system working in L-Band, at the center frequency of $1.325 \mathrm{GHz}$ with a bandwidth of $150 \mathrm{MHz}$ is required. The antenna is realized in planar technology and thereby a microstrip patch is considered for the single element, due to its conformability, ease of manufacture, integration and low cost. The aperture coupled slot technique for the antenna feeding provides versatility to implement the dual polarization characteristic $[2,3]$.

Microstrip antennas are typically narrowband due to their resonant nature (3-4\% bandwidth). In order to overcome such disadvantage and to achieve the required bandwidth of $150 \mathrm{MHz}$, around $12 \%$, some broadband techniques have to be applied such as the stacked-patch configuration or the use of additional foam layers [4,5]. The design of the single antenna element combines and enhances some of the later concepts in order to achieve the required performance. For the standard side looking SAR configuration an antenna main beam pointing at 45 degree from broadside radiation, with a side lobe level suppression of $20 \mathrm{~dB}$ and a minimum at nadir (perpendicular to airplane flight direction) are required. 
In order to satisfy such requirements, an antenna array with an amplitude and phase taper is designed. Figure 1 shows the antenna mount carried by the DLR Do-228 aircraft with the current L-Band antenna.

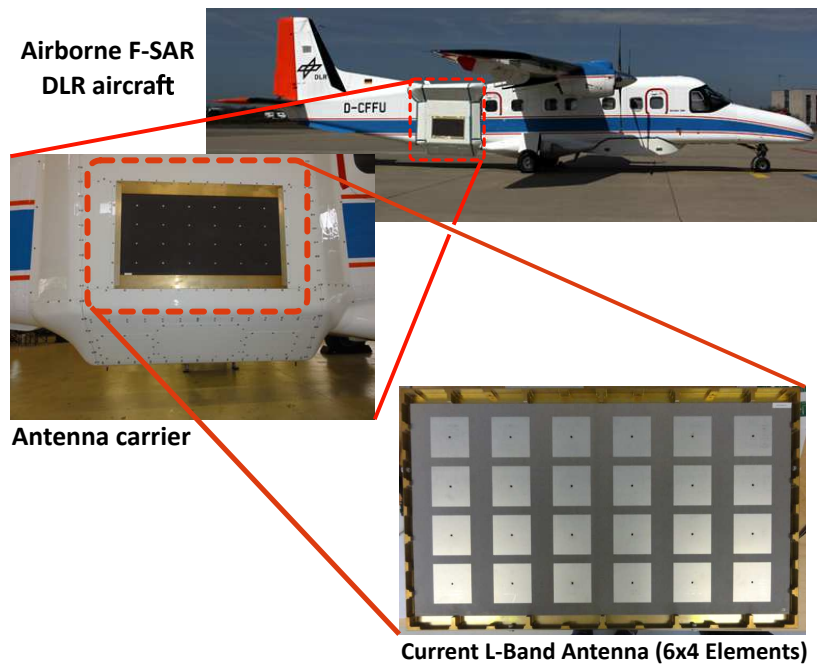

Fig. 1. View of the DLR aircraft and antenna carrier. Current L-Band planar array with $6 \times 4$ elements.

\section{ANTENNA SINGLE ELEMENT}

\section{A. Design Steps}

For the design of the single antenna element, a microstrip double patch antenna in a stacked configuration fed by an aperture slot is chosen.

In order to enhance the broadband behaviour of the antenna, a dual stacked patch is implemented. Both patches resonate at slightly different frequencies (different patch lengths), thereby providing a higher bandwidth.

The lower patch is electromagnetically coupled by an aperture (etched slot in the antenna ground plane) that at the same time couples the upper patch. This aperture slot is also coupled by a microstrip line from which the antenna is fed.

The required dual polarization operation implies the use of rectangular patches due to its both resonant lengths, two orthogonal aperture slots and their corresponding microstrip lines. The design of the single element involves a multi-layer structure with a radiating and feeding part, as it can be seen in Figure 2.

Two substrate layers with high dielectric constant (Rogers RT/Duroid 6006) were used to reduce the patch size. These layers are also needed mechanically to support the patches. The top substrate placed above the patch increases not only the effective permittivity and thus reducing the patch size, but it also serves as antenna radome or protective layer. The properties of each layer are displayed in Table 1.

Each microstrip feed line is placed at different substrate heights (FR4) in order to provide a high isolation between the two feed ports. Figure 3 provides a view of the antenna feeding mechanism.

The H-shape slot improves the electromagnetic coupling between the slot and the lower patch [6]. It also helps to reduce
Table 1. Antenna layers.

\begin{tabular}{|c|c|c|c|}
\hline Name & Substrate & $\varepsilon_{r}$ & Thickness \\
\hline Substrate Patch 1 & Rogers RT/Duroid 6006 & 6.15 & $1.27 \mathrm{~mm}$ \\
\hline Foam 1 & Rohacell HF 51 & 1.06 & $18 \mathrm{~mm}$ \\
\hline Substrate Patch 2 & Rogers RT/Duroid 6006 & 6.15 & $1.27 \mathrm{~mm}$ \\
\hline Foam 2 & Rohacell HF 51 & 1.06 & $4 \mathrm{~mm}$ \\
\hline Feed Substrate 1 & FR4 Epoxy & 4.4 & $1.27 \mathrm{~mm}$ \\
\hline Feed Substrate 2 & FR4 Epoxy & 4.4 & $1.27 \mathrm{~mm}$ \\
\hline Foam 3 & Rohacell HF 51 & 1.06 & $5 \mathrm{~mm}$ \\
\hline
\end{tabular}

the electrical length of the slot, which is useful to optimize the available space. Both slots are shifted with a small offset. The values of each line length are measured from its respective slot center. The antenna geometrical values of Figure 3 are listed in Table 2.

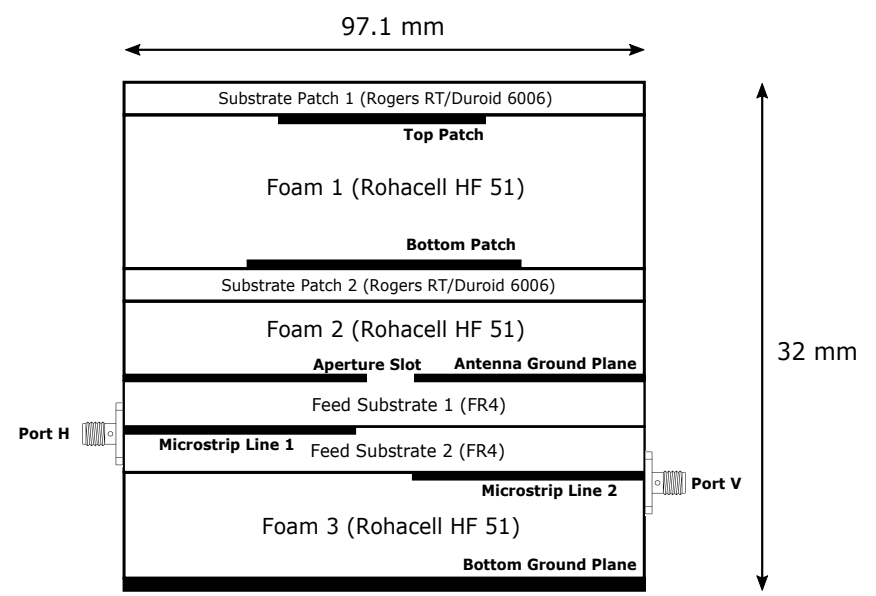

Fig. 2. Multi-layer structure of a single patch element.

This multi-layer structure is enclosed within four electric walls. This cavity-box provides a higher isolation between array elements by preventing the transmission of surface waves, thereby reducing the inter-element coupling. The inclusion of these walls also reduces the antenna bandwidth due to the resonant nature of the cavity. In the presented design, a bandwidth of around $15 \%$ is achieved.

Without this cavity-box configuration, the stacked patch technique and the use of thick foam layers can provide bandwidths up to $20-30 \%$. However this leads to a strong coupling between elements of $-12 \mathrm{~dB}$ or greater, above all in the antenna E-plane, which is not acceptable. This coupling reduction considering the later array case, where the elements are separated less than $0.5 \lambda$, is essential, due to the fact that it is desired to take advantage of the increased number of elements in order to shape more accurately the radiation pattern.

These design steps lead to a very compact design that allow exploiting the limited available space for the antenna installation by reducing the single element size and increasing the overall number of elements.

The design procedure and later optimization was performed with HFSS, part of the ANSYS Electromagnetics Suite 19.1. 
Table 2. Antenna geometrical values.

\begin{tabular}{|c|c||c|c|}
\hline Top patch length & $59.6 \mathrm{~mm}$ & Bottom patch length & $65.6 \mathrm{~mm}$ \\
\hline Top patch width & $58.8 \mathrm{~mm}$ & Bottom patch width & $63.8 \mathrm{~mm}$ \\
\hline Line 1 length & $18.5 \mathrm{~mm}$ & Line 2 length & $28.5 \mathrm{~mm}$ \\
\hline Line 1 width & $1.85 \mathrm{~mm}$ & Line 2 width & $4.15 \mathrm{~mm}$ \\
\hline Slot 1 length & $40 \mathrm{~mm}$ & Slot 2 length & $37 \mathrm{~mm}$ \\
\hline Slot 1 width & $2.5 \mathrm{~mm}$ & Slot 2 width & $2.5 \mathrm{~mm}$ \\
\hline Edge 1 length & $11 \mathrm{~mm}$ & Edge 2 length & $11 \mathrm{~mm}$ \\
\hline Edge 1 width & $2.5 \mathrm{~mm}$ & Edge 2 width & $2.5 \mathrm{~mm}$ \\
\hline Slot offset 1 & $17 \mathrm{~mm}$ & Slot offset 2 & $10 \mathrm{~mm}$ \\
\hline
\end{tabular}

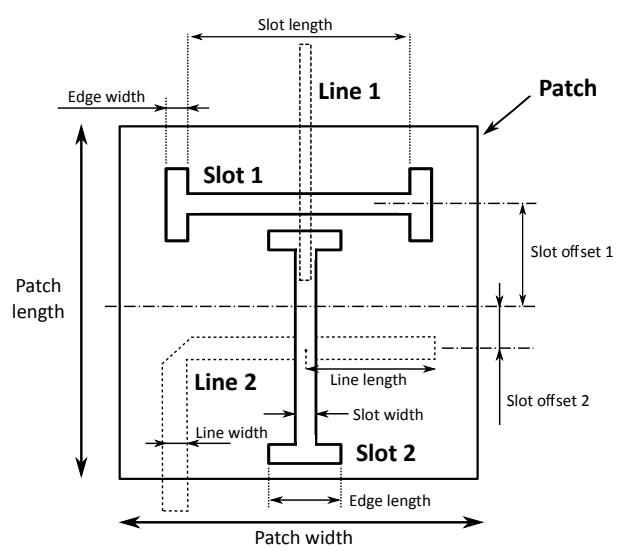

Fig. 3. Aperture coupling layout.

\section{B. Manufactured Single Element}

As mentioned before, the single antenna was constructed in a cavity-box configuration with electric walls, in order to reduce the inter-element interaction between antennas (Fig. 4). This construction allows easily connecting the antennas to build a linear array by attaching intermediate plates of different thicknesses in order to analyze the coupling between elements. The measurements meet very well the simulation results as it can be seen in the S-Parameters of Figure 5 and the radiation pattern of Figure 6 . The center frequency of the real antenna is slightly shifted due to fabrication tolerances. The radiation pattern of the manufactured single element, measured at the DLR Compact Test Range of the Microwaves and Radar Institute [7], shows an outstanding agreement with the simulation. The measured gain of polarization $\mathrm{H}(6.1 \mathrm{dBi})$ and $\mathrm{V}(6 \mathrm{dBi})$ differs only approximately $0.3 \mathrm{~dB}$ from the simulated values. The simulated efficiency of the antenna is around $93 \%$.

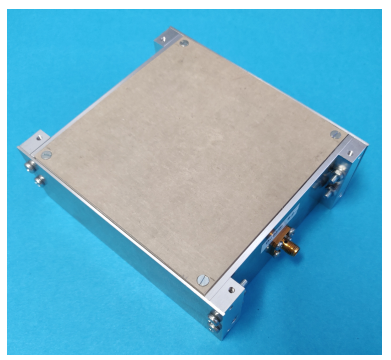

(a)

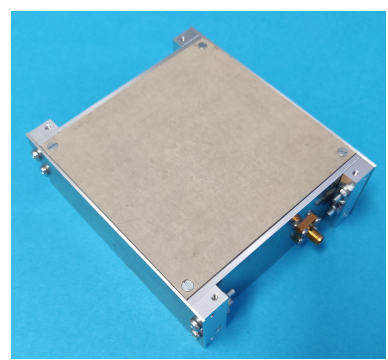

(b)
Fig. 4. Manufactured single element antenna. Each view shows one feed port, which are placed opposite: (a) Polarization H; (b) Polarization V.

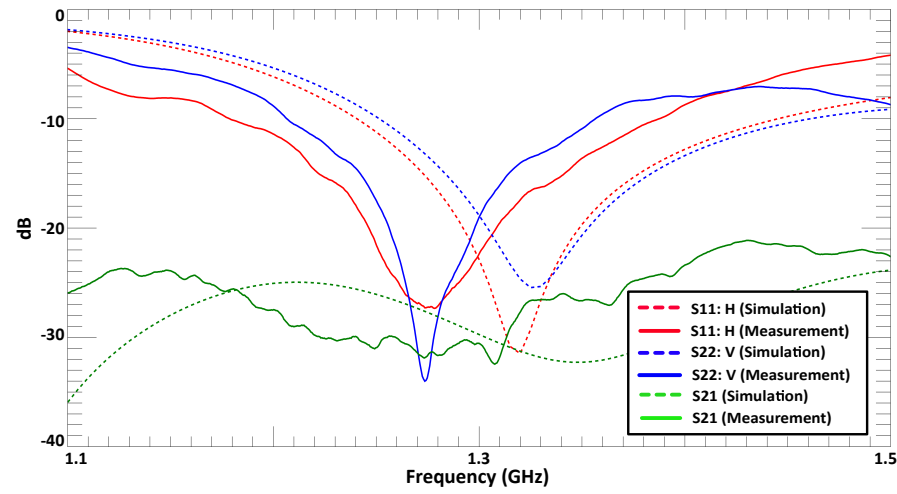

Fig. 5. Simulated and measured return loss and port isolation for each polarization. Port 1 corresponds to horizontal and port 2 to vertical.

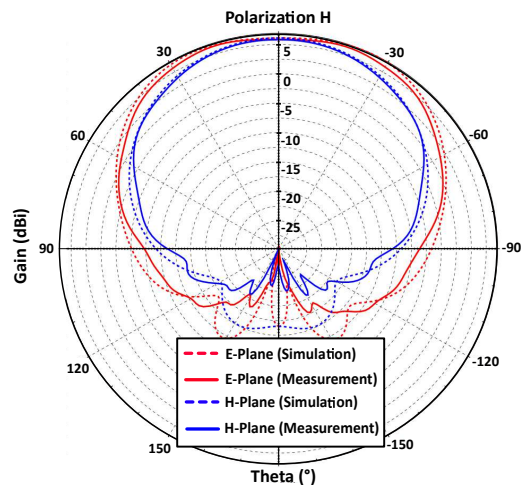

(a)

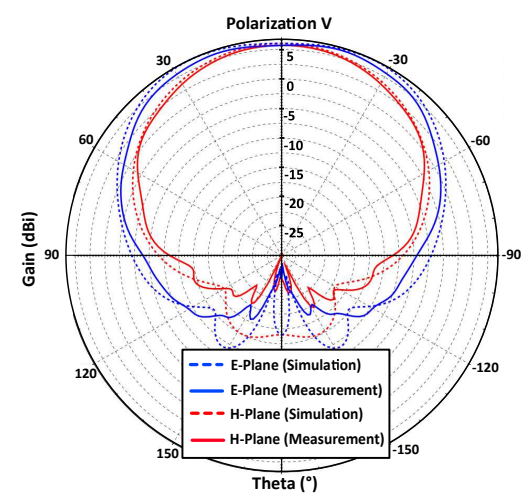

(b)

Fig. 6. Simulated and measured radiation pattern: (a) Polarization $\mathrm{H}$; (b) Polarization V.

\section{ANTENNA ARRAY}

\section{A. Linear Array Design}

The presented single element concept is extended to build a linear array in order to fulfill the system requirements and to test the feasibility of the antenna system.

It is well known that, in order to avoid the appearance of grating lobes, an inter-element spacing of $0.5 \lambda$ is required. However, due to space constraints within the antenna carrier, a smaller distance has to be used. Considering the whole available space, an array of five elements with an inter-element spacing of around $0.48 \lambda$ can be built, by using intermediate 
plates of $10 \mathrm{~mm}$ thickness, as it can be seen in Figure 7. For this configuration, a mutual coupling of $15 \mathrm{~dB}$ is obtained, which allows taking advantage of the increased number of antenna elements to shape the radiation pattern as required.

\section{B. Manufactured Array}

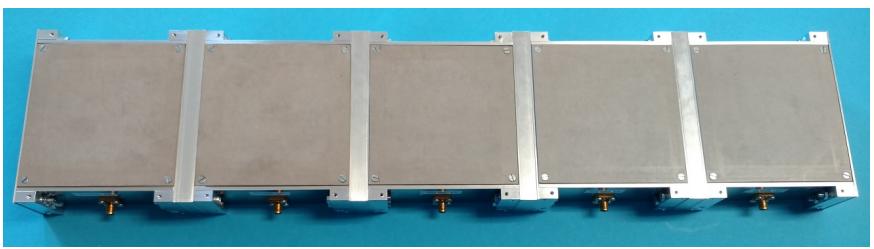

Fig. 7. Manufactured array of 5 elements, interconnected with 4 intermediate plates of thickness $10 \mathrm{~mm}$.

To achieve the $45^{\circ}$ tilted beam a phase shift of $120^{\circ}$ between elements was applied by using different self made cable lengths connected to each antenna. To reduce the side lobe level an amplitude taper with triangular distribution is used and implemented, in this prototype case, by means of a power splitter and different attenuators.

Figure 8 shows again a really good agreement between the measured radiation pattern of the manufactured array and the simulated value.

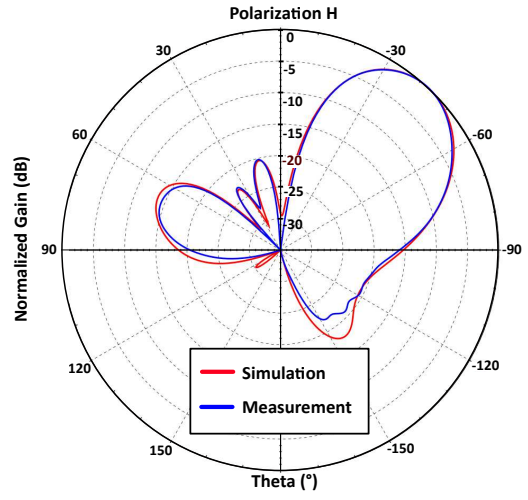

(a)

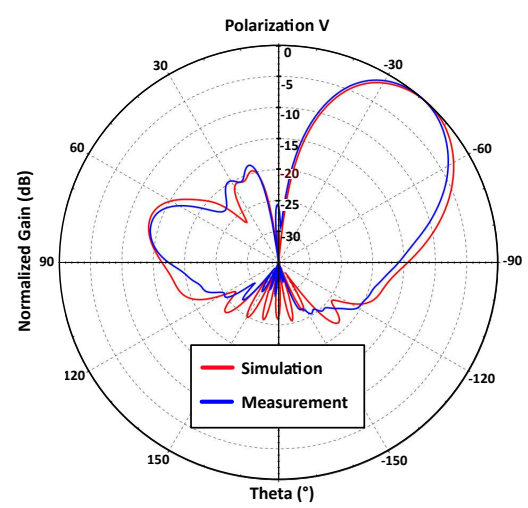

(b)

Fig. 8. Simulated and measured normalized pattern of the array: (a) Elevation cut for polarization H; (b) Elevation cut for polarization V.
The array provides around $10.5 \mathrm{dBi}$ gain and a cross polarization level below $-25 \mathrm{~dB}$. The system presents a good stability in frequency for the desired bandwidth.

This antenna design can be extended in order to build a planar array with up to 40 elements, with an expected gain of $20 \mathrm{dBi}$ for side looking configuration.

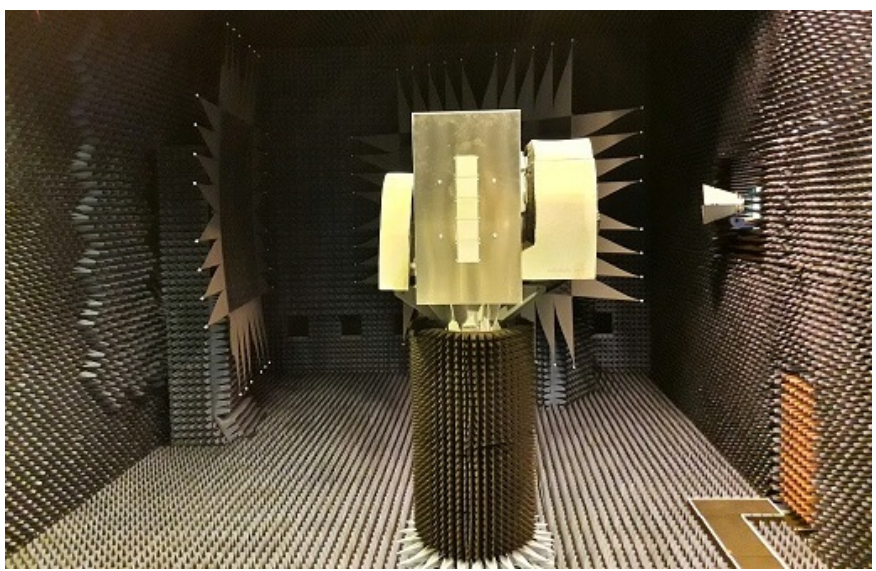

Fig. 9. DLR's Compact Test Range facilities during the array measurement.

\section{CONCLUSION}

The new antenna concept for the next generation of the German Aerospace Center L-Band airborne SAR sensor was presented and validated. The radiation properties of the antenna are maximized by increasing the number of single elements, in comparison with the current configuration.

The future work involves extending the presented antenna concept to design the final planar array and feed network for the final airborne sensor. With the new design a planar antenna of up to 40 elements $(8 \times 5)$ can be achieved which implies, in comparison with the current antenna $(6 \times 4)$, an increment of up to 16 more antenna elements $(66 \%)$.

\section{ACKNOWLEDGMENT}

We would like to thank Evonik Industries for the delivery of sample foam layers Rohacell $\mathrm{HF} 51$ required for the manufacturing of the prototype antenna.

\section{REFERENCES}

[1] R. Horn, M. Jaeger, M. Keller, M. Limbach, A. Nottensteiner, M. Pardini, A. Reigber and R. Scheiber, "F-SAR - Recent Upgrades and Campaign Activities" in 18th International Radar Symposium (IRS), June 2017.

[2] D. M. Pozar, "Microstrip antennas", IEEE Proc., vol. 80, pp. 79-91, Jan. 1992.

[3] D. M. Pozar, "A review of Aperture Coupled Microstrip Antennas: History, Operation, Development and Applications", May 1996.

[4] W. S. T. Rowe, and Rod. B. Waterhouse, "Investigation Into the Performance of Proximity Coupled Stacked Patches", IEEE Trans. Antennas Propag, vol. 54, June 2006.

[5] M. Bugaj and M. Wnuk, Bandwidth Optimization of Aperture-Coupled Stacked Patch Antenna, IntechOpen, Chapter 2, 2013.

[6] D. M. Pozar, and S. D. Targonski "Improved coupling for aperture coupled microstrip antenna" IEEE Electron Device Lett., vol. 27, no.13, June 1991.

[7] M. Limbach, B. Gabler, R. Horn, A. Reigber, "DLR-HR Compact Test Range facility" in European Conference on Antennas and Propagation (EuCAP), March 2009. 